\title{
Anisotropia do manto litosférico por re-polarização de ondas SKS: implicações na evolução tectônica do Brasil central
}

Elias Martins G. Prado, José Eduardo P. Soares, Reinhardt A. Fuck, Laboratório de Estudos da Litosfera, Instituto de Geociências, Universidade de Brasília (LabLitos/IG/UnB)

Copyright 2012, SBGf - Sociedade Brasileira de Geofísica

Este texto foi preparado para a apresentação no V Simpósio Brasileiro de Geofísica, Salvador, 27 a 29 de novembro de 2012. Seu conteúdo foi revisado pelo Comitê Sácnico do $V$ SimBGf, mas não necessariamente representa a opinião da SBGf ou de seus associados. É proibida a reprodução total ou parcial deste material para propósitos comerciais sem prévia autorização da SBGf.

\section{Resumo}

An array of six broadband seismographic stations was installed in central Brazil, covering the transition between the Tocantins Province and the Amazon paleoplate along two NW-SE profiles, with the aim to imaging the structure of the lithosphere in the region. Using SKS phases from teleseismic event recordings, we were able to determine the anisotropy directions of the lithospheric mantle. Almost all the events came from null directions, remaining an ambiguity. The ambiguity was resolved by analyzing the regional structures and the geology. The results suggest that the lithospheric mantle beneath the region has undergone regional effort to NW-SE direction, producing pronounced foliation in NE-SW direction. This direction is coincident with the direction of Transbrasiliano Lineament and the regional geological trend.

\section{Introdução}

A Província Tocantins é resultado da convergência entre as paleoplacas São-Franciscana, Amazônica e Paranapanema durante a orogenia Brasiliana no Neoproterozoico. O norte da província, localizado entre os cratons São Francisco e Amazônico, apresenta trend geológico para NE-SW, coincidente com a direção do alto gravimétrico Bouguer regional $(200 \mathrm{~km}$ de largura e $700 \mathrm{~km}$ de comprimento) localizado no Brasil central. A analise integrada dos dados de refração sísmica profunda com os dados gravimétricos, mostra que o alto gravimétrico representa um corredor, onde o manto Neoproterozoico se encontra mais raso, denso, quente e provavelmente menos depletado do que os mantos Paleoproterozoicos vizinhos, relacionados aos cratons Amazônico e São Francisco. Com intuito de determinar a anisotropia do manto litosférico nesta região através do estudo de fases SKS, foram analisados telessismos registrados em seis estações broadband pertencentes a rede sismográfica para estudos tectônicos do LabLitos(UnB), localizadas no limite oeste do alto gravimétrico do Brasil central (Figura 1). As estações estão organizadas ao longo de dois perfis NW-SE. Um próximo a latitude $13^{\circ} \mathrm{S}$ onde se encontram as estações RET8 e RET9 (uma estação se encontra acima do gradiente gravimétrico e a outra a noroeste). O segundo perfil, próximo da latitude $12^{\circ} \mathrm{S}$, contem as estações RET2, RET3 e RET4, instaladas acima do gradiente gravimétrico, a oeste, e a leste. Ao norte, próximo ao complexo máfico-ultramáfico acamadado de Porto
Nacional, se encontra a estação RET1, instalada acima do alto gravimétrico.

A anisotropia no manto superior pode ser resultado de uma deformação antiga ou de uma deformação atual, que esta em andamento. A deformação decorrente de um evento de orogênese passado produz uma trama no manto superior, que pode continuar estável após o relaxamento termal do orógeno, chamada comumente de anisotropia congelada (Ben Ismail \& Mainprice, 1988; Founch \& Rondenay, 2006; Nicolas \& Christensen, 1987; Plomerová et al., 2008; Savage, 1999; Vauchez \& Nicolas, 1991). A movimentação e deformação atual do manto astenosférico, relacionada com o movimento das placas tectônicas, também pode produzir uma orientação dos cristais de olivina. Esta movimentação é a principal geradora da anisotropia em manto litosférico que se encontra abaixo de crosta oceânica (Conrad et al., 2007; Tommasi, 1998; Wolfe \& Silver, 1998).

A região de estudo se localiza em terrenos tectonicamente estáveis, com a estruturação principal formada durante o Neoproterozóico/Cambriano, logo a anisotropia do manto superior nesta região provavelmente se trata de uma anisotropia congelada, produzida durante a aproximação das paleoplacas Amazônica e São-Franciscana.

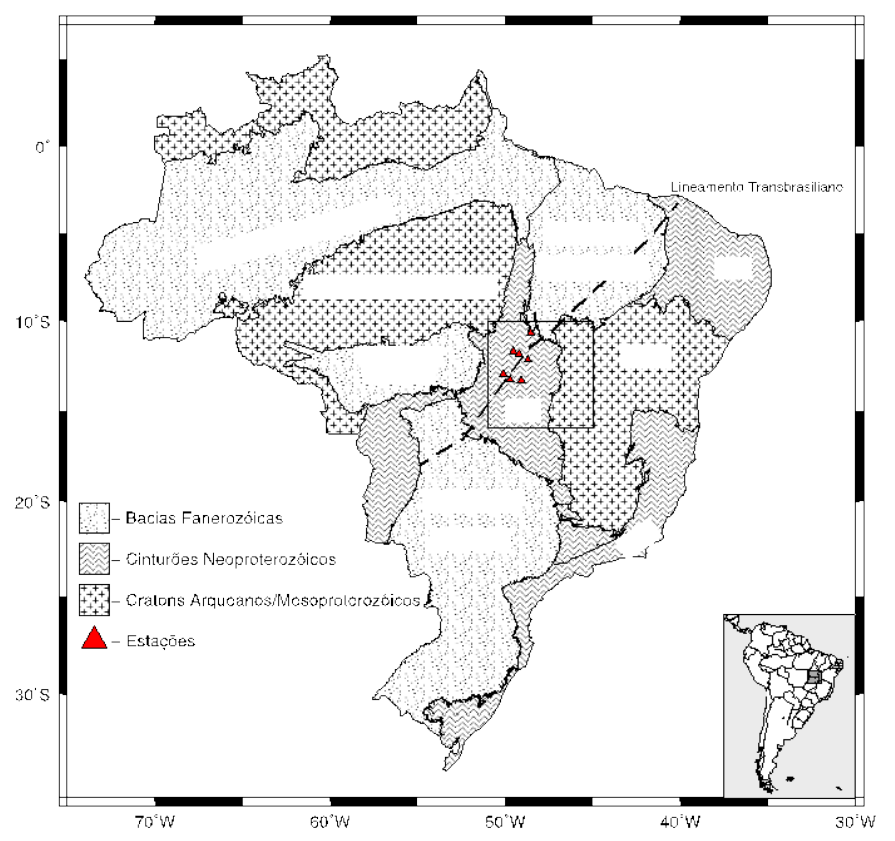

Figura 1 - Localização das estações sismográficas estudadas. As texturas mostram as principais províncias geológicas e estruturais do Brasil (Almeida et al., 1977, 1984). (CSF - Cráton São Francisco; PT - Província Tocantins; PM - Província Mantiqueira; PB - Província Borborema) 


\section{Metodologia/ Problema Investigado}

Comumente os estudos de anisotropia do manto superior são baseados na analise das componentes radia $\mathrm{R}$ e transversal T (sistema de coordenadas ZRT, Z - vertical), assumindo uma incidência vertical das ondas cisalhantes. Neste trabalho utilizamos o programa SplitLab (Wüstefeld et al., 2008), que utiliza o sistema de coordenadas LQT ( $L$ - longitudinal, paralelo a direção de propagação da onda, $\mathrm{T}$ - transversal e $\mathrm{Q}$ - radial, normal ao plano $\mathrm{L}-\mathrm{T}$ ), procurando pela direção de polarização da fase rápida no plano Q-T perpendicular ao plano de trajetória da fase cisalhante L-Q. Uma rotação do sistema de coordenadas no plano Q-T por um angulo $\Psi$, e uma diferença de tempo $\delta$ t entre as componentes da onda cisalhante, produzem os parâmetros de separação. A direção da fase rápida também pode ser definida pelo azimute $\Phi$ (medido a partir do Norte no plano horizontal E-N).

Para remover o efeito de separação das ondas cisalhantes, afim de encontrar os parâmetros programa SplitLab utiliza simultaneamente três técnicas diferentes, sendo elas o método de rotação-correlação (Bowman \& Ando, 1987), o método da mínima energia (Silver \& Chan, 1988) e o método de autovalor (Silver \& Chan, 1991). Todas as três técnicas realizam uma busca em grid para determinar os parâmetros melhor removem o efeito de separação da fase SKS, isto é, linearizam o movimento de partícula nos planos E-N e Q-T. Como um critério para a melhor linearização, a técnica de rotação-correlação usa o aumento do coeficiente de cross-correlação entre a forma de onda nas componentes radial $\mathrm{Q}$ e transversal $\mathrm{T}$. A técnica de mínima energia procura pela mínima energia ou integral de correlação CTT na componente transversal $(E T=$ CTT). A técnica de autovalor procura pelo menor autovalor $\lambda$ da matriz de correlação. Embora os métodos de rotação-correlação e autovalor sejam comumente usados para se determinar os parâmetros de separação de ondas, frequentemente eles podem fornecer resultados instáveis ou falsos quando comparados com o método da mínima energia (Luděk Vecsey et al., 2008).

Quando a polarização inicial da fase SKS (paralela ao backazimuth) é paralela ou perpendicular a direção de anisotropia do manto a fase não é re-polarizada, gerando resultados nulos ou falsos (Savage, 1999). Teste sintéticos mostram que as técnicas de rotação-correlação e mínima energia apresentam comportamentos característicos quando isto acontece. Próximo de backazimuths nulos o método de rotação-correlação apresenta valores de $\delta$ t sistematicamente baixos e direções de anisotropia desviando em até $45^{\circ}$ das direções reais. O método da mínima energia é relativamente estável em backazimuths nulos, porém as direções de anisotropia podem desviar $90^{\circ}$ da direção real e os valores de $\delta$ t apresentam grande dispersão (Wustefeld \& Bokelmann, 2007) (Figura 3 e 4).

Resultados (Arial Bold, 9)

A analise dos resultados mostra que os eventos estudados apresentam backazimuths em direções de nulos, o que gera uma ambiguidade nos resultados. Em todas as estações o método de mínima energia apresentou duas direções principais de anisotropia, uma variando de N40E a N60E e outra variando de N30W a $\mathrm{N} 50 \mathrm{~W}$, porem as direções NE foram as mais populosas na maioria das estações. Nas estações em que a direção NW foi a mais populosa, a direção NE foi escolhida por ser a mais provável direção de polarização rápida. O atraso $\delta$ entre as fases rápida e lenda no método de mínima energia apresentaram grande dispersões, e no método de rotação-correlação ficaram todos muito próximos de zero por se tratarem de direções nulas, o que aumenta o erro na determinação do סt. Apesar do grande erro na determinação dos atrasos foram estimados atrasos variando de 1s a 1.7s (Tabela $1 \mathrm{e}$ Figura 2).

\section{Discussão e Conclusões}

A direção de anisotropia e o retardo entre as fases sugerem que o manto litosférico tenha sido submetido a esforço regional de direção NW-SE, produzindo pronunciada foliação na direção NE-SW. Essa direção é coincidente com a direção do Lineamento Transbrasiliano, do alto gravimétrico do Brasil central, dos lineamentos magnéticos regionais e com o trend geológico regional. A convergência dos resultados para estações sismográficas situadas tanto no domínio da paleoplaca Amazônica quanto no da Província Tocantins, no interior e a oeste do Lineamento Transbrasiliano, sugerem que a anisotropia do manto litosférico nessa região tenha sido formada pela convergência de NW para SE da paleoplaca Amazônica, durante o amálgama final de Gondwana ocidental no Neoproterozóico. A comprovação de possível expressão do Lineamento Transbrasiliano no manto litosférico carece de resultados adicionais obtidos em regiões afastadas de grandes movimentações tectônicas.

Tabela 1 - Resultados de Anisotropia mostrando a direção de anisotropia $\Phi$ e 0 atraso

\begin{tabular}{ccc} 
Estação & $\Phi(\stackrel{\circ}{ })$ & $\delta \mathrm{t}(\mathrm{s})$ \\
\hline RET1 & $48 \pm 10$ & $1.7 \pm 0.5$ \\
RET2 & $56 \pm 10$ & $1 \pm 0.5$ \\
RET3 & $56 \pm 6$ & $1.2 \pm 0.5$ \\
RET4 & $60 \pm 8$ & $1.2 \pm 0.5$ \\
RET8 & $46 \pm 4$ & $1.2 \pm 0.3$ \\
RET9 & $45 \pm 5$ & $1.1 \pm 0.3$
\end{tabular}




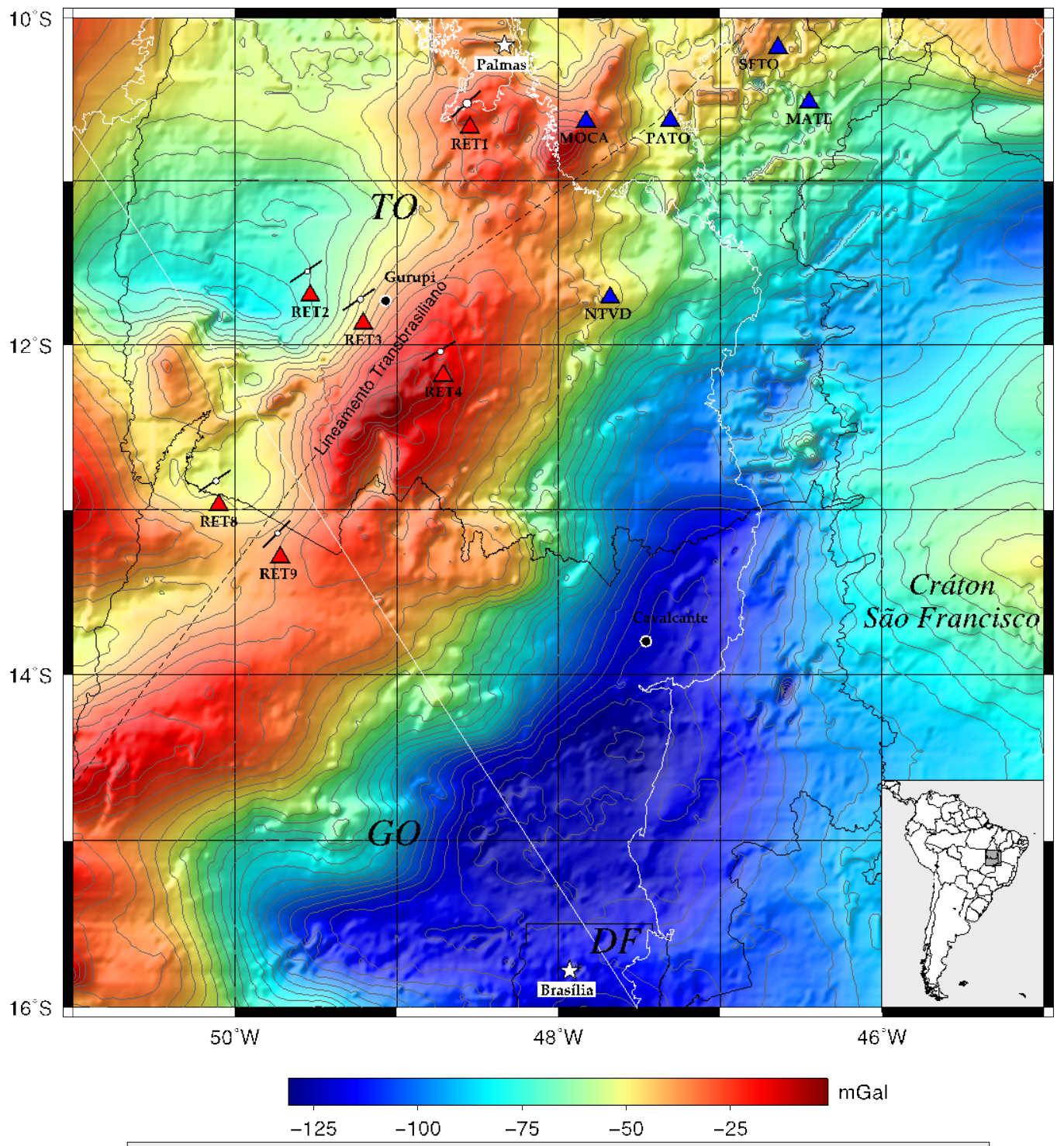

\begin{tabular}{|c|c|c|c|}
\hline \multicolumn{4}{|c|}{$\begin{array}{c}\text { Direções de Anisotropia do Manto Litosférico entre } \\
\text { as Paleoplacas Amazônica e São Fransiscana }\end{array}$} \\
\hline \multicolumn{4}{|c|}{$\begin{array}{l}\text { Projeção: Geográfica } \\
\text { Datum: WGS-84 }\end{array}$} \\
\hline \multicolumn{4}{|c|}{$100 \mathrm{~km}$} \\
\hline \multicolumn{4}{|c|}{$\begin{array}{l}\text { Mapa de Anomalia Bouguer regional ao fundo, filtrado } \\
\text { para comprimentos de onda maiores que } 50 \mathrm{~km} \text {. Intervalo de contorno } 5 \mathrm{mGal}\end{array}$} \\
\hline \multicolumn{4}{|l|}{ Legenda: } \\
\hline $\mid-\Phi$ & $\circ-1 \mathrm{~s} \delta \mathrm{t}$ & $\circ-1,5 \mathrm{~s} \delta \mathrm{t}$ & $-2 \mathrm{~s} \delta \mathrm{t}$ \\
\hline
\end{tabular}

Figura 2 - Direções de anisotropia do manto litosférico entre as Paleoplacas Amazônica e São Franciscana, as estações estudadas neste trabalho estão indicadas como triângulos vermelhos. 

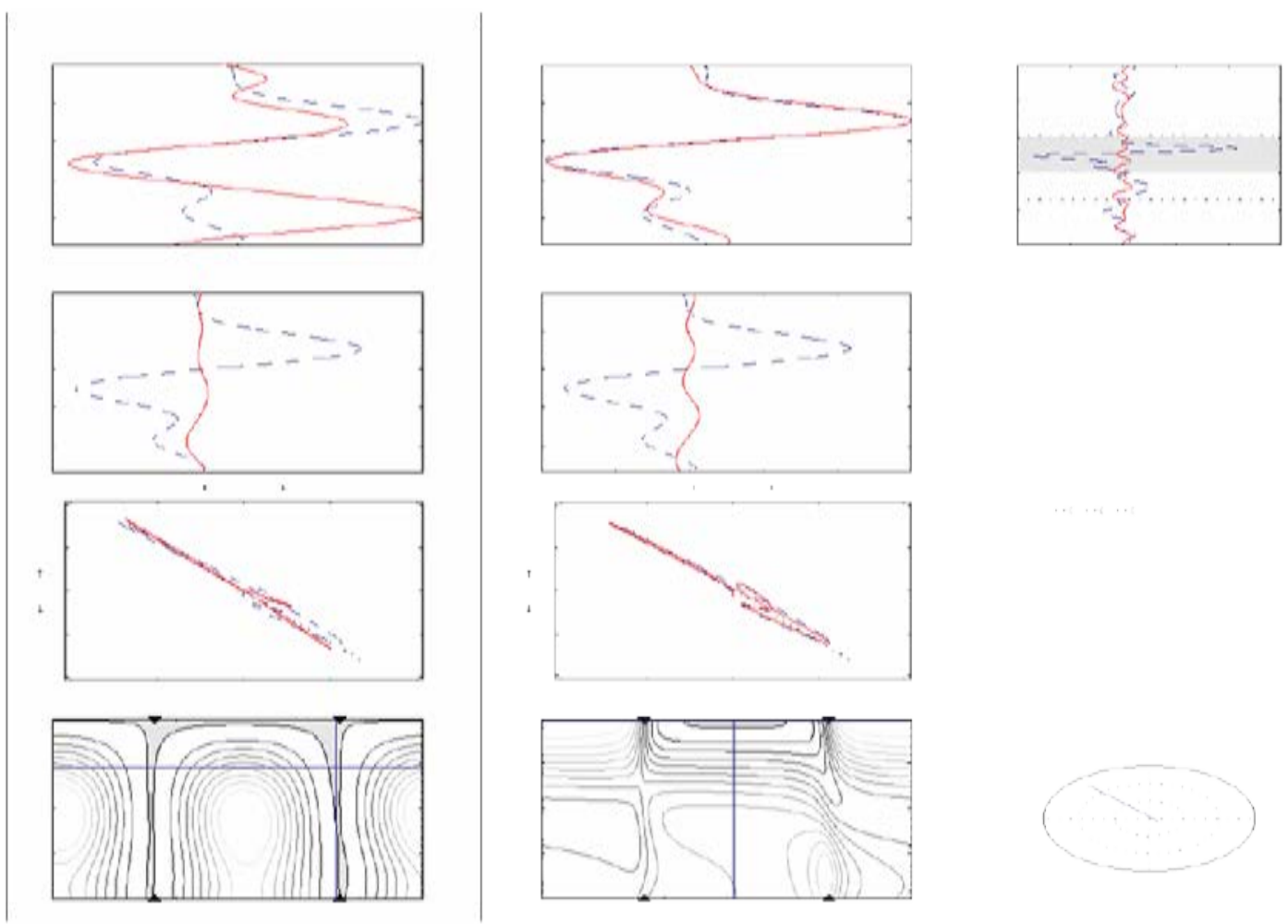

Figura 4 - Exemplo de um resultado obtido pelo programa SplitLab para a estação RET9. O resultado apresenta características de um resultado nulo típico.

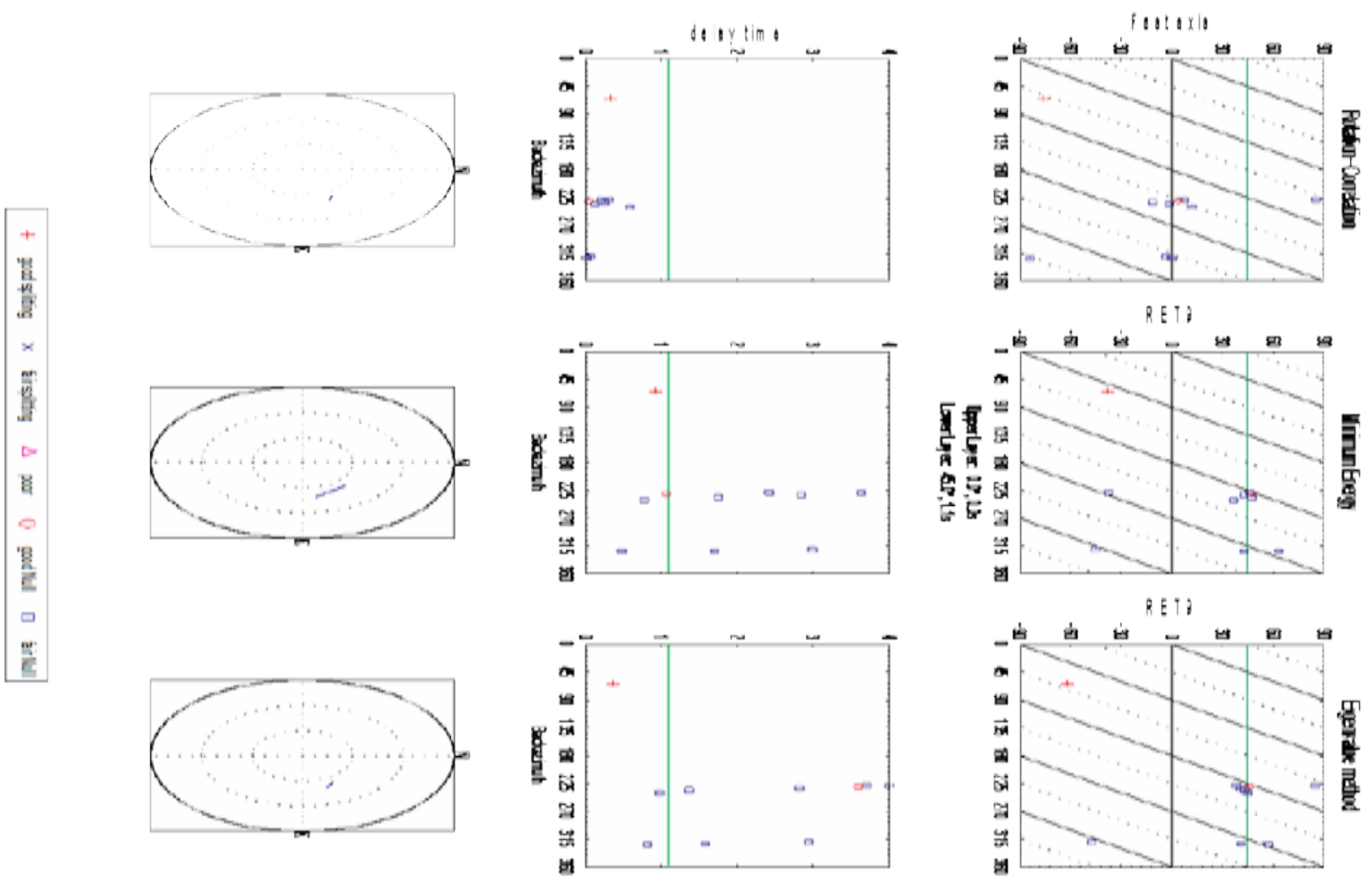

Figura 3 - Resultados da estação RET9, acima direções de anisotropia e abaixo o atraso de tempo $\delta$ t. 


\section{Agradecimentos}

O Autor agradece José Eduardo P. Soares e Reinhardt A. Fuck pelo apoio, sugestões e revisão, e o LabLitos/UnB que forneceu toda a estrutura para realização do trabalho.

\section{Referências}

Ben Ismail, W., \& Mainprice, D. (1988). An olivine fabric database: Anoverview of upper mantle fabrics and seismic anisotropy. Tectonophysics, 296, 145-157.

Bott, M. H. P., 1982. Stress based tectonic mechanisms at passive continental margins. In: Scrutton, R. A. (Ed.), Dynamics of Passive Margins. AGU Geodynamic Series, Washington (DC), USA, vol. 6: 147-153.

Bowman, J. R., \& Ando, M. (1987). Shear-wave splitting in the upper-mantle wedge above the Tonga subduction zone. Geophys. J. R. Astron. Soc., 88, 25-41.

Conrad, C. P., Behn, M. D., \& Silver, P. G. (2007). Global mantle flow and the development of seismic anisotropy: Differences between the oceanic and the continental upper mantle. Journal of Geophysical Research, 112.

Founch, M. J., \& Rondenay, S. (2006). Seismic anisotropy beneath stable continental interiors. Physics of the Earth and Planetary Interiors, 158, 292-320.

Nicolas, A., \& Christensen, N. I. (1987). Formation of anisotropy in upper mantle peridotites - A review, in Funchs, K., and Froidevaux, C., eds., Composition, Structure and Dynamics of the LithosphereAsthenosphere System: American Geophysical Union, 111-123. Washington, D.C.

Plomerová, J., Frederiksen, A. W., \& Park, J. (2008). Seismic anisotropy and geodynamics of the lithosphereastenosphere system. Tectonophysics, 462, 1-6.

Savage, M. K. (1999). Seismic anisotropy and mantle deformation: What have we learned from shear wave spliting? Review of Geophysics, 37, 65-106.

Silver, P. G., \& Chan, W. W. (1988). Implications for continental structure and evolution from seismic anisotropy. Nature, 335, 34-39.

Silver, P. G., \& Chan, W. W. (1991). Shear Wave Splitting and Subcontinental Mantle Deformation. Geophysics. Res., 96(B10), 16429-16454.

Wolfe, C., \& Silver, P. G. (1998). Seismic anisotropy of oceanic upper mantle: Shear wave splitting methodologies and observations. Journal of Geophysical Research, 103, 749-771.
Vecsey, Luděk, Plomerová, J., \& Babuška, V. (2008). Shear-wave splitting measurements - Problems and solutions. Tectonophysics, 462(1-4), 178-196. Elsevier B.V. doi:10.1016/j.tecto.2008.01.021.

Wüstefeld, A., Bokelmann, G., Zaroli, C., \& Barruol, G. (2008). SplitLab: A shear-wave splitting environment in Matlab. Computers \& Geosciences, 34(5), 515-528. doi:10.1016/j.cageo.2007.08.002

Wustefeld, a., \& Bokelmann, G. (2007). Null Detection in Shear-Wave Splitting Measurements. Bulletin of the Seismological Society of America, 97(4), 1204-1211. doi:10.1785/0120060190. 
V Simpósio Brasileiro de Geofísica 